\title{
Bioinspirated algorithm for multi-criterial problem solution of production schedule optimization
}

\author{
Olga Chengar ${ }^{1}$, Victoria Shevchenko ${ }^{1, *}$, and Dmitrii Voronin ${ }^{1}$ \\ ${ }^{1}$ Federal State Autonomous Educational Institution of Higher Education «Sevastopol State University», \\ 299053, Sevastopol, Russian Federation
}

\begin{abstract}
In the article the multi-criterial problem of production schedule synthesis of the machine-building enterprise has been considered. The proposed bioinspirated ant colony algorithm can be used to increase the quality the machining area functioning by considering simultaneously several factors that influence the efficiency of technological equipment loading.
\end{abstract}

\section{Introduction}

One of the most important problems in the field of operational production management is the task of planning of the process equipment usage. Practical experience shows that in a real production situation a rational, rather than an optimal plan, usually is been created. In this regard, an actual approach is to ensure the multi-criterion usage allowing the playback of plan options analysis based on a group of criteria. The real optimization problem is usually solved for several criteria. In addition, there is the contradiction between production deadlines compliance and the equipment effective usage. It can be greatly weakened by setting the priorities that form a partially ordered sequence of manufactured items instead of timing regulation usage. These priorities can be quickly changed based on the current needs. In a practical production environment it is necessary to create a work schedule taking into account several criteria simultaneously. The need of a compromise search, that takes into account several conflicting criteria, forms the urgency of the considering problem.

In multi-criteria optimization several functions are used to estimate the efficiency of the proposed solution $[1,2]$. This significantly complicates the problem and requires the use of specialized approaches. In general, the problem of multi-criteria optimization can be formulated as follows (1):

$$
\max (\min )\left\{z_{1}=f_{1}(x), \ldots, z_{q}=f_{q}(x)\right\}, g_{i}(x) \leq 0, x \geq 0, i=1,2, \ldots, m .
$$

where $f_{q}(x)$ - criterion function; $q$ - number of criteria; $g_{i}(x)$ - task limit; $m$ - number of limitations.

\footnotetext{
* Corresponding author: shevchenko-vika@,mail.ru
} 
According to the Pareto's dominance principle, the potential solutions of the multicriteria problem can be classified into dominated and non-dominated [2]. The $x$ solution is called dominated, if there is $y$ solution, that is not worse than $x$ by all the criteria, thus the following conditions are met for all optimizable functions $f_{i}(i=1, \ldots, q)$ :

- $f_{i}(x) \leq f_{i}(y)$ for all $1 \leq i \leq k$ when the function $f_{i}$ is maximized ;

- $\quad f_{i}(x) \geq f_{i}(y)$ for all $1 \leq i \leq k$ when the function $f_{i}$ is minimized.

If the solution is not dominated by any other solution, then it is called non-dominant or optimal in the sense of Pareto.

Scientific publications analysis of modern methods [3-6], used for solving of the multicriteria optimization problem, shows that promising approaches are mostly based on the idea of Pareto set approximation using evolutionary algorithms. Fitness function is used primarily in the selection of individuals for further evolution and here quality characteristics of one individual relative to other individuals are important. Definition of fitness function for solving a specific problem with evolutionary algorithms is critical to its effectiveness. Fundamental feature is not in the use of the bio-based algorithms. The main idea is to form the rules of the fitness functions formation in such a way that the movement of individuals' population corresponds to the direction of the Pareto set [5]. The evolution of these individuals can be proceed according to laws that differ from the laws used in evolutionary algorithms, for example, it can be done according to the laws of ant population motion as it is done in the ant algorithm. Thus, the task of multi-criteria optimization based on the so-called "population" Pareto-approximation methods (for example, the method of ant colonies) becomes urgent.

\section{Research problem formulation}

In the works [7-9] the problem of synthesis of a suboptimal production schedule has been considered. For its solution, a certain numerical function $F(G)$ (criterion function) has been implemented. It is defined on all $G(i)$ graphs and assigns correspondence between each graph $G(i)$ and a certain number $F\left(G_{i}\right)$. In this case, the best count matches $F(G)$ function's extremum. Thus, the problem is to construct a graph that satisfies all the formulated constraints, on which the function $F(G)$ reaches its extreme value [8].

Evaluating the effectiveness of the resulting production schedules can be made by one of the criteria:

- minimizing the violation;

- maximizing the average load factor of flexible manufacturing modules;

- minimization of the cycle time manufacturing parts;

- minimizing the time changeovers;

- not completed production minimization;

- number of using flexible manufacturing modules minimization.

Justification of effectiveness criterion choice is a responsible and non-trivial task. The difficulty is that the various optimality criteria are often contradictory and, thus, optimization by one criterion leads to a quality deterioration by another criterion. To solve this problem, it is expedient to use an integrated criterion, for example, the sum of particular criteria with some expertly assigned coefficients. However, this approach also requires a serious justification, first, because of the considerable arbitrariness in the choice of the integrated criterion structure and the assignment of the coefficients.

Ant algorithms base on the use of a multitude of potential solutions in various directions of global search, because they do not impose any requirements on the form of objective functions and limitations [7]. Therefore, multi-criteria optimization uses not single, but multiple variants of paths for each ants' population, moreover, all of them are optimal in the sense of Pareto and the central issue is the formation of the objective function. The 
performed analysis [10] has shown efficiency of the weighted sum method, which determines the weight of each criterion included in the objective function. This approach is one of the most popular and natural development of classical optimization methods, where the "general" objective function is constructed from separate objective functions in the form of a weighted sum (2):

$$
F(x)=\sum_{i=1}^{k} w_{i} f_{i}(x), \sum_{i=1}^{k} w_{i}=1
$$

where $w_{i}$ - the weight of each criterion included in the objective function; $k$ - number of criteria.

For the formulated multi-criterial problem of production schedule synthesis of the machine-building enterprise it is advisable to use the Pareto-optimization method based on the "directed" ant algorithm with adaptive weights.

\section{Pareto optimization method, based on "directed" ant algorithm with adaptive weights}

As a rule, in a real production situation it is problematic to determine the gradation of efficiency criteria in the order of increasing importance, and, accordingly, to allocate priorities and assign the necessary weighting factors for the integrated objective function. Thus, the use of adaptive weights in the integrated optimality criterion reflects the tendency of the alternating search direction that is more suitable for finding the front of solutions.

Thus, the essence of the multi-criteria approach based on the "directed" ant algorithm with adaptive weights can be represented by the following algorithm (fig. 1).

So for each objective, denoted as $f_{k}(x)$, its weight $w_{k}$ is determined (4). The scalar value of the new objective function is then calculated by summing the weighted values $q$ of optimality criteria. As for the parallel search multiple solutions weights are not fixed, then it is possible to expand the front in all directions for the "directed" ant algorithm.

In the presented algorithm, at each iteration, a set of solutions based on the "directed" ant algorithm is formed according to a certain optimization criterion. Further, for the studied solutions, the maximum and minimum extremal points are determined in the space of given criteria (3):

$$
z^{+}=\left\{z_{1}^{\max }, z_{2}^{\max }, \ldots, z_{q}^{\max }\right\}, z^{-}=\left\{z_{1}^{\min }, z_{2}^{\min }, \ldots, z_{q}^{\min }\right\}
$$

For each criterion, the maximum and minimum values are determined as follows (4):

$$
z_{k}^{\max }=\max \left\{f_{k}(x) \mid x \in P\right\}, z_{k}^{\min }=\min \left\{f_{k}(x) \mid x \in P\right\}, k=1,2, \ldots, q
$$

where $z_{k}^{\min }$ и $z_{k}^{\max }$ - minimum and maximum values for the $\mathrm{k}$-th objective according to the specified criterion;

$q$ - the number of optimality criteria;

$P$ - a set of solutions by a given criterion.

As a result, the obtained hyperplane contains all the current solutions and two extremal points define it. These points are updated at each iteration. In this case, the adaptive weight of the $\mathrm{k}$-th objective is determined by the relation (5).

$$
w_{k}=\frac{1}{z_{k}^{\max }-z_{k}^{\min }}, k=1,2, \ldots, q
$$

For each objective, the average weighting factors are established based on the selected effectiveness criteria, which are normalized within the group (6). 


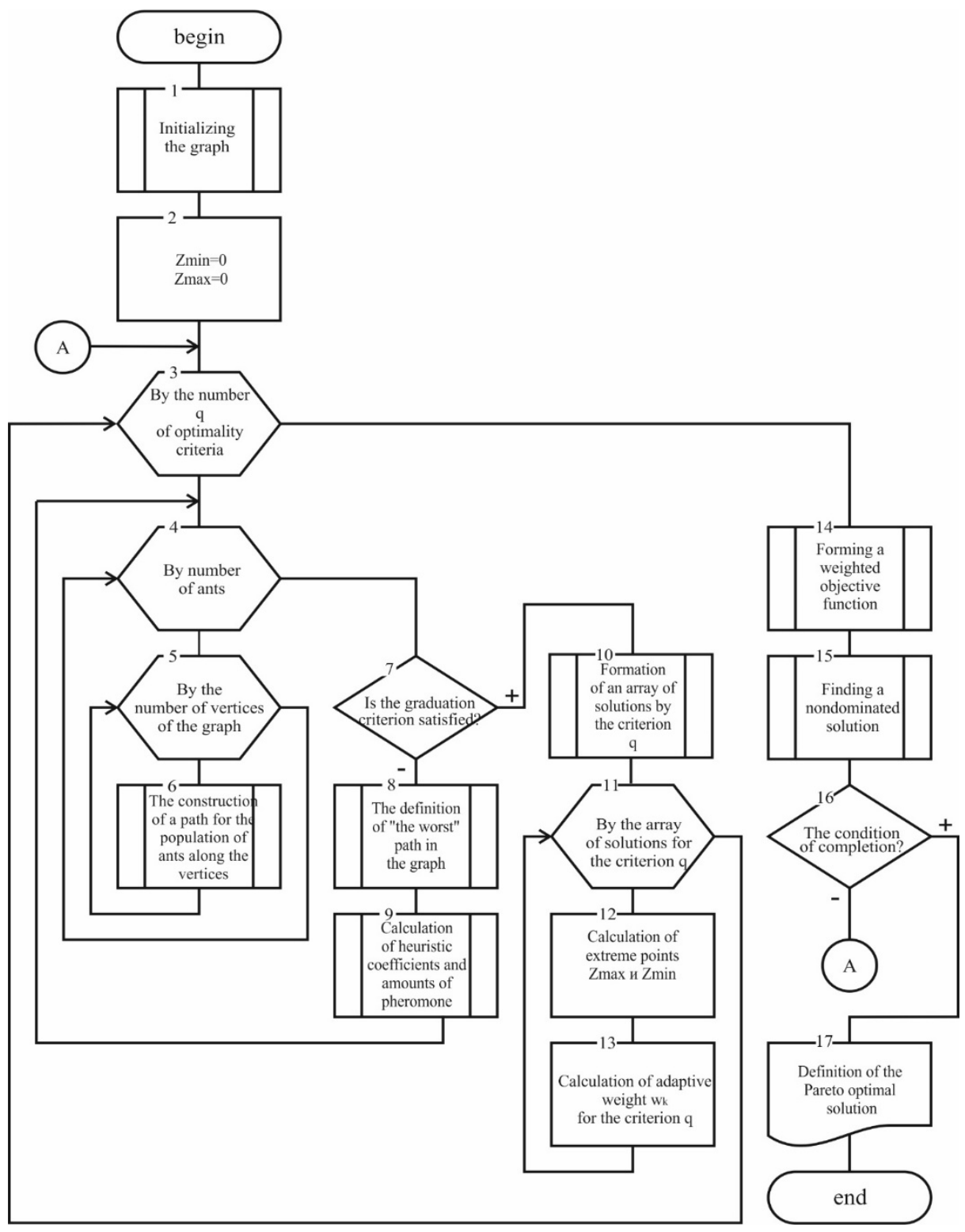

Fig. 1. "Directional" ant algorithm with adaptive weights.

$$
w_{k}^{\text {norm. }}=\frac{w_{k}}{\sum_{i=1}^{q} w_{i}}, k=1,2, \ldots, q
$$

Then, at each iteration, the weighted objective function is determined according to the following relation (7).

$$
Z(x)=\sum_{i=1}^{q} w_{k}^{n o r m} \cdot\left(f_{k}(x)-z_{k}^{\min }\right)
$$


All the considered Pareto solutions lie in the space $Z$ and during the process of finding a solution for each population of artificial agents, the hyperplane consistently approaches the positive (or negative) ideal point. This method allows to adjust the weights of the objective function and directs the search for solutions in the right direction.

As each of the optimality criteria can have its own solution accuracy $[9,12]$, which is achieved with a different number of iterations $\left(n_{t}\right)$, for determining $n_{t}$ experimental research was conducted. The maximum value from all number of iterations under different criteria is the stop criterion for the multi-criteria problem solving process.

\section{Experimental study of the proposed method}

Analysing the efficiency criteria it is possible to single out a number of objectives suitable for studying the multicriteria task of planning of technological equipment usage. Criteria selection was based on the following statements:

- optimization objectives do not contradict each other;

- optimization is to be carried out in one direction (minimization or maximization);

- the requirements of the actual production situation are to be met.

Based on the above, the following combinations of performance criteria were selected:

- to minimize the manufacturing order deadline violation under the minimum manufacturing item cycle duration;

- to minimize the manufacturing order deadline violation under the minimum changeover time of the equipment;

- to minimize the manufacturing parts duration under the minimum changeover time of the equipment;

- to minimize the manufacturing order deadline violation under the minimum manufacturing item cycle duration and the minimum changeover time of the equipment.

Figure 2 shows the result of the two-criterion problem: to minimize the manufacturing order deadline violation under the minimum manufacturing item cycle duration.

Since optimization performs according two criteria, then there can occur a situation when there are more than one solution, it is called the Pareto front. Therefore, in the actual production situation, it is urgent to find only one "good" solution, but in a rather short time. In this connection, it is necessary to select all control parameters for each production area experimentally even during the stage of algorithm debugging $[10,11]$.

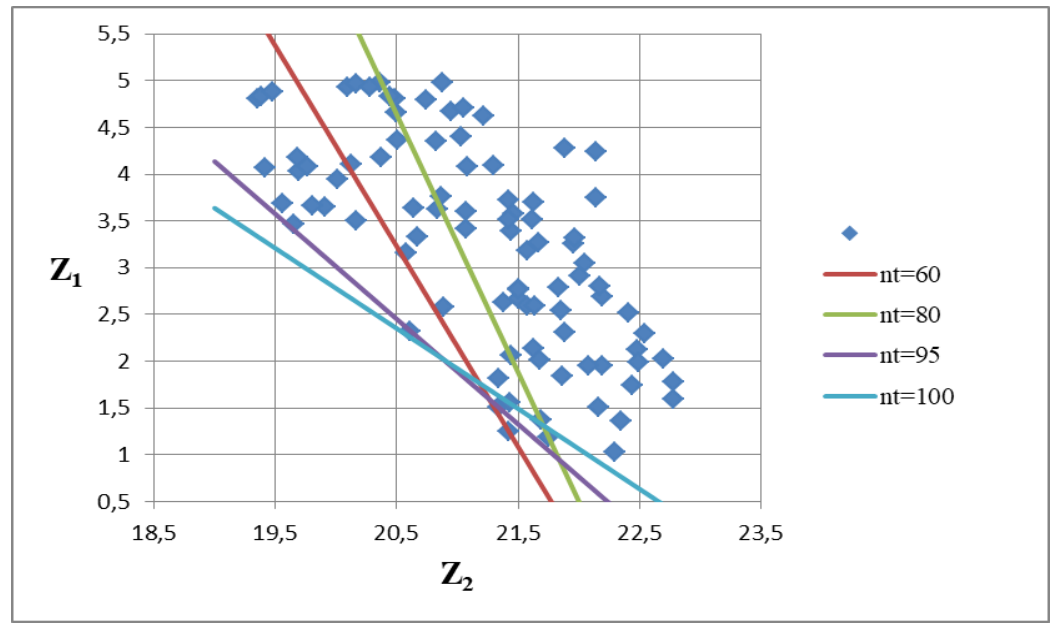

Fig. 2. The result of Pareto optimization of a two-criterion problem. 
The considered month-long production program consists of 200 items that can be of 3 different types. Experimentally it has been found out that maximum number of iterations that are necessary to obtain a suboptimal Pareto solution is $n_{t}=100$. For greater clarity, the presented problem is mapped in the criteria space in order to approximate the solution to the Pareto-optimal. Bold points denote all the dominant points obtained in the course of the algorithm. Direct lines indicate a consistent approximation of the adaptive line to nondominant solutions based on the number of ants' populations. The solution of the considered problem is the conditional minimum point. It can be found as the lower intersection point of the admissible region with the adaptive straight line, built on a given number of iterations.

Similar actions were carried out for other options of the selecting criteria $[14,15]$, including a three-criterion problem. The properties inherent in ant algorithms contribute to the their effective application in solving multi-criteria problems because ant algorithms are based on the use a multitude of potential solutions in the global search in various directions and do not impose any requirements on the form of objective functions and constraints. The results of the solution of the proposed test tasks allow to conclude that the modification of the ant colony method and the Pareto dominance concept are successful - the points are located almost evenly and over the whole set of criteria, the adaptive straight line moves successively to the non-dominant points, thus approaching the Pareto-optimal solution.

\section{Conclusion}

The aim of the conducted research was to take into account several factors affecting production planning. It has been clarified that the Pareto optimization method based on the bioinspiral algorithm with adaptive weights allows to search through the entire solution space for each population of artificial agents and to choose optimal variants (in the sense of Pareto) from them. This can be used to increase the quality the machining area functioning.Experimental research $[13,15]$ of the proposed method allow to assert, that introduction of additional performance criteria significantly complicates the problem of suboptimal solutions choice, and however, the method's effectiveness doesn't reduce significantly. So, according to various criteria of effectiveness, the discrepancy between the results of single-criterion and two-criteria problems are within $1 \div 3 \%$; single-criterion and three-criteria tasks - within $6 \div 8.5 \%$, which is acceptable.

This work was supported by the Russian Foundation for Basic Research (projects № 15-29-07936, № 1847-920007, № 18-47-920005).

\section{References}

1. K. Deb. Multi-objective optimization using evolutionary algorithms UK: Wiley, (2001)

2. E. Zitzler, K. Deb, L. Thiele. Evolutionary Computation Vol. 8(2), P. 173-195, (2000).

3. D. Moor, D. Mukhlisullina. Science and education: electronic scientific and technical publication Vol. 4, P. 123-129, (2010).

4. S. Mostaghim, J. Teich. In: Swarm Intelligence Symposium, P. 26- 33, (2003).

5. V. Guliashki, H. Toshev, Ch.Korsemov Problems of Engineering Cybernetics and Robotics Vol. 60, P. 42 - 54, (2009).

6. V. Podinovsky, V. Nogin. Pareto-optimal solutions of multicriteria problems. RU: Moscow, (2007). 
7. V. Nogin. Decision-making in a multicriteria environment: a quantitative approach. RU: Moscow, (2005).

8. D. Ashlock Evolutionary Computation for Modeling and Optimization. Springer, (2006).

9. I. Sobole, R. Statnikov The choice of optimal parameters in problems with many criteria. RU: Moscow, (2006).

10. O. Chengar Problems of Information Technology. Bulletin of Kherson National Technical University, Vol. 1(46). P. 212-217. (2013).

11. O. Chengar E. Savkova. Journal of East Ukrainian National University. 13 (167), P. 239-245, (2011).

12. Y. Skobtsov, A. Sekirin, S. Zemlyanskaya, O. Chengar, V. Skobtsov, S. Potryasaev. Advances in Intelligent Systems and Computing, vol.466, P.453-462, (2016).

13. Y. Skobtsov, O. Chengar, V. Skobtsov, A. Pavlov. Advances in Intelligent Systems and Computing, vol.573, P.456-465, (2017).

14. O. Chengar, E. Vladimirova, E.Savkova, N. Sapozhnikov. Matec WEB of Conferences, vol.129, P. 03013, (2017).

15. O. Chengar, E. Mashchenko, D. Zamorenova, A. Phedosov. Matec WEB of Conferences, vol.129, P. 03015, (2017).

16. J. Ryu, S. Kim. Proceedings of the 2009 Winter Simulation Conference (WSC), P. 623-633, (2009).

17. Y. Skobtsov, D. Speransky, Evolutionary computation (The National Open University "INTUIT", 2015)

18. A. A. Ambarcumjan, A. S. Hadeev. Analysis of the functionality of maintenance management systems and equipment repair. Problemy upravlenija 6, pp. 2-12, (2005). 